\title{
Low-Dose Aspirin Acetylates Cyclooxygenase-1 in Human Colorectal Mucosa: Implications for the Chemoprevention of Colorectal Cancer
}

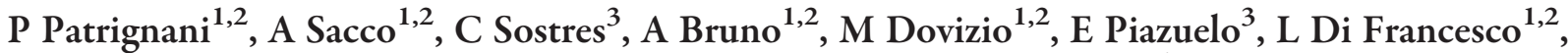

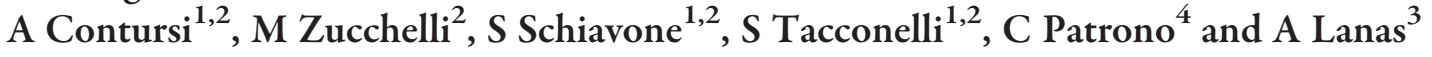

The mechanism of action of low-dose aspirin in the prevention of colorectal cancer (CRC) remains largely hypothetical. We aimed to compare the effects of low-dose aspirin (100 mg/day for 7 days) given to 40 individuals undergoing CRC screening on the extent of cyclooxygenase (COX)-1 acetylation at serine-529 (AceCOX-1), in blood platelets vs. colorectal mucosa, at 7 (group 1) and $24 \mathrm{~h}$ (group 2) after dosing. A significantly $(P<0.01)$ lower \%AceCOX-1 was detected in colonic and rectal mucosa (average 64\%) vs. platelets (average $75 \%$ ) in both groups. This effect was associated with an average $46 \%(P<0.01)$ and 35\% $(P<0.05)$ reduction in prostaglandin $(P G) E_{2}$ levels and phosphorylated $S 6(p-S 6)$ levels, respectively. Rectal mucosal levels of $p$-S6/S6 significantly $(P<0.01)$ correlated with $\mathrm{PGE}_{2}$. These findings demonstrate that low-dose aspirin produces long-lasting acetylation of COX-1 and downregulation of p-S6 in human colorectal mucosa, an effect that may interfere with early colorectal carcinogenesis.

Study Highlights

\section{WHAT IS THE CURRENT KNOWLEDGE ON THE TOPIC? \\ $\checkmark$ A large body of evidence supports a chemopreventive effect of low-dose aspirin against CRC; however, its cellular target(s) remains largely hypothetical. \\ WHAT QUESTION DID THIS STUDY ADDRESS? \\ $\square$ To what extent and for how long does low-dose aspirin acet- ylate COX-isozymes in normal colorectal mucosa as compared to blood platelets in subjects undergoing CRC screening.}

WHAT THIS STUDY ADDS TO OUR KNOWLEDGE $\checkmark$ We demonstrate that low-dose aspirin persistently inhibits COX-1 activity expressed in colorectal mucosa, thus restraining $\mathrm{PGE}_{2}$ biosynthesis and $\mathrm{S} 6$ phosphorylation, an important component of colorectal tumorigenesis.

HOW THIS MIGHT CHANGE CLINICAL PHARMACOLOGY OR TRANSLATIONAL SCIENCE

$\square$ Our findings of two distinct drug targets of low-dose aspirin pharmacodynamics may help design dose-finding studies addressing the optimal dosing regimen for CRC prevention. Moreover, it may offer an investigational paradigm for studies of other cancer sites potentially targeted by aspirin chemoprevention.
A large body of evidence supports a chemopreventive effect of low-dose aspirin against colorectal cancer (CRC). ${ }^{1,2}$ However, the mechanism of action remains largely hypothetical.

Inhibition of ubiquitous cyclooxygenase (COX)-dependent prostanoid production is necessary and sufficient to explain the multifaceted clinical readouts of aspirin pharmacodynamics. ${ }^{3,4}$ Although other mechanisms have been proposed to explain its chemopreventive effect, COX-isozymes remain the only drug target inhibitable at low micromolar aspirin concentrations in vitro and following oral administration of low doses in vivo, and for which a crystal structure has been obtained explaining target function modification by the drug. ${ }^{6}$
An inhibitory effect on COX-2-dependent inflammation represents a widely accepted mechanism to explain the chemopreventive effect of aspirin and other nonsteroidal antiinflammatory drugs (NSAIDs). ${ }^{7}$ However, whether COX-2 is expressed in healthy colorectal mucosa remains controversial, and whether low-dose aspirin can inhibit mucosal COX-2 activity in vivo has not been previously investigated.

Constitutive expression of COX-1 in the gastrointestinal (GI) mucosa is generally considered to subserve housekeeping functions, such as mucosal cytoprotection, through prostaglandin $(\mathrm{PG}) \mathrm{E}_{2}$ production, ${ }^{4}$ but its role in colorectal carcinogenesis has only been explored in a murine model of intestinal polyposis. ${ }^{8}$ It

\footnotetext{
${ }^{1}$ Section of Cardiovascular and Pharmacological Sciences, Department of Neuroscience, Imaging and Clinical Science, “G. d'Annunzio" University, Chieti, Italy; ${ }^{2} \mathrm{CeSI}-\mathrm{MeT}$ (Centro Scienze dell' Invecchiamento e Medicina Traslazionale), “G. d'Annunzio” University, Chieti, Italy; ${ }^{3}$ University Hospital LB, Aragon Health Research Institute (IISAragon), CIBERehd, University of Zaragoza, Zaragoza, Spain; ${ }^{4}$ Institute of Pharmacology, Catholic University School of Medicine, Rome, Italy. Correspondence: P Patrignani (ppatrignani@unich.it) 
has been proposed that in early stages of intestinal tumorigenesis, enhanced colorectal $\mathrm{PGE}_{2}$ biosynthesis might occur via constitutively expressed COX-1 in association with the suppression of the PG-catabolizing enzyme, 15-prostaglandin-dehydrogenase (15-PGDH), before the induction of COX-2. ' However, the occurrence of this mechanism in vivo remains to be demonstrated.

Micromolar concentrations of aspirin cause irreversible inhibition of COX-1 activity by acetylating a serine 529 (Ser529) residue near the catalytic site of the enzyme. ${ }^{6,10,11}$ Although previous studies have reported reduced GI levels of $\mathrm{PGE}_{2}$ in response to low-dose aspirin, ${ }^{12-14}$ a potential contribution of platelet $\mathrm{PGE}_{2}$ inhibition cannot be excluded because of platelet activation during and after tissue biopsy. No previous study has directly examined the extent and duration of COX-1 acetylation in colorectal mucosa by low-dose aspirin and its impact on local $\mathrm{PGE}_{2}$ production.

Thus, we designed a study to address the following related questions: 1) which COX-isozyme is expressed by apparently healthy colorectal mucosa, and provides the primary source of $\mathrm{PGE}_{2}$ production in subjects undergoing CRC screening; 2) to what extent is colorectal mucosa COX-1 acetylated by low-dose aspirin as compared to platelet COX-1;3) is acetylation of COX-1 in the colorectal mucosa a reversible process because of de novo synthesis of the enzyme by nucleated cells, and is it accompanied by measurable reduction in $\mathrm{PGE}_{2}$ production; and 4 ) is there a detectable reduction in the expression of proteins involved in the pathophysiology of CRC. Among the different signaling pathways of intestinal tumorigenesis, we studied the impact of low-dose aspirin on the extent of phosphorylation of ribosomal protein S6. It may occur at Ser235/236 by $\mathrm{p} 70^{\text {S6K }}$, which is directly activated by the mammalian target of rapamycin $(\mathrm{mTOR})^{15}$ and by other kinases, including PKA. ${ }^{16,17}$ The phosphorylation of S6 increases the affinity of the $40 S$ ribosomal subunit to a subclass of mRNAs, and thus promotes their efficient translation, and seems to play a potential role in regulating cell growth capacity. ${ }^{18}$

\section{RESULTS}

We performed a short-term, randomized study of 40 subjects undergoing CRC screening who received enteric-coated (EC)-aspirin 100 mg/day (Adiro; supplied by Bayer-Schering Pharma, Berlin, Germany) for 7 days before the colonoscopy. The inclusion and exclusion criteria are detailed in the Supplementary Methods. Consenting subjects were randomly allocated to group $1(n=20)$ or group $2(n=20)$, and peripheral blood and tissue samples (biopsies of the rectal-sigmoidal segment and left and right colon) were collected at 7 and $24 \mathrm{~h}$ after the last dose of aspirin, respectively (Figure 1). The two groups were characterized by similar demographic and clinical characteristics, including concomitant pharmacologic treatments (Supplementary Tables S1, S2).

\section{Assessment of plasma levels of acetylsalicylic acid (ASA) and salicylic acid (SA) and biomarkers of platelet inhibition}

At $7 \mathrm{~h}$ after the last dose of aspirin (group 1), plasma levels of ASA and SA averaged $39.4 \pm 19.3$ and 1,500 $\pm 300 \mathrm{ng} / \mathrm{mL}$, respectively. In contrast, in group 2 ( $24 \mathrm{~h}$ after dosing), both ASA and SA were undetectable (data not shown).

We assessed the effects of low-dose aspirin on the extent and duration of platelet COX-1 acetylation at Ser529 (AceCOX-1). As shown in Table 1 and Figure 2, at 7 h (group 1) the extent of platelet COX-1 acetylation averaged $74.8 \pm 8.1 \%$ and was not significantly different from the mean value found at $24 \mathrm{~h}$ after dosing (group 2: $75.2 \pm 8.8 \%$ ). This effect was associated with virtually complete suppression of platelet COX-1 activity (>97\%), as reflected by $\mathrm{TXB}_{2}$ production during whole blood clotting (serum $\mathrm{TXB}_{2}$ ), ${ }^{19,20}$ in both groups (Table 1). Aspirin caused comparable inhibition of platelet function, as assessed by the PFA-100 diagnostic system with collagen/epinephrine test cartridge, ${ }^{20} 7$ and $24 \mathrm{~h}$ after dosing (Table 1). Aspirin also caused similar reductions in the urinary excretion of TX-M, an index of TXA $_{2}$ biosynthesis in vivo ${ }^{21,22}$ in both groups (Table $\mathbf{1}$ ).

\section{Expression of proteins involved in $\mathrm{PGE}_{2}$ biosynthesis and catabolism and $\mathrm{PGE}_{\mathbf{2}}$ levels in normal rectal biopsies}

We studied the expression levels of COX-1, COX-2, mPGES-1 (microsomal PGE synthase-1), and 15-PGDH in the rectal mucosa of subjects of both groups, at baseline and after aspirin treatment (Figure 3a-c). Mucosal expression of COX-1 was assessed by LC-MS/MS ${ }^{20,23}$; aspirin did not affect total protein concentration (AceCOX-1+COX-1) in either group (Figure 3a).

No detectable expression of COX-2 was found by western blot analysis; the results of representative biopsies collected in three different individuals are shown in Figure 3b. mPGES-1 and 15-PGDH levels, assessed by western blot, are reported in Figure 3b,c. In both groups, aspirin treatment did not significantly affect the levels of these proteins. Small changes in 15-PGDH levels were detected in some individuals after aspirin treatment, both in group 1 (Figure $\mathbf{3 b}, \mathbf{c}$ ) and group 2 (Figure $3 \mathbf{c}$ ).

$\mathrm{PGE}_{2}$ is a major prostanoid produced in the colorectal mucosa. ${ }^{24}$ Before aspirin administration, similar levels of $\mathrm{PGE}_{2}$ were measured in rectal mucosal biopsies collected at baseline in groups 1 and $2(5.8 \pm 2.9$ and $6.2 \pm 2.9 \mathrm{pg} / \mu \mathrm{g}$ protein, respectively) (Figure 3d). Treatment with low-dose aspirin caused a significant $(P<0.01)$ reduction in $\mathrm{PGE}_{2}$ levels by $43 \%$ in group 1 and $50 \%$ in group 2 (Figure 3d), suggesting a persistent inhibitory effect of aspirin on mucosal COX-1 activity up to $24 \mathrm{~h}$ after dosing.

\section{Assessment of COX-1 acetylation in biopsies of normal colorectal mucosa}

We used a previously described liquid chromatography/tandem mass spectrometry (LC-MS/MS) technique to measure the extent of COX-1 acetylation at Ser $529,{ }^{20,23}$ in proteolyzed biopsies of the intestinal mucosa. As shown in Figure 2, at 7 and $24 \mathrm{~h}$ after aspirin (groups 1 and 2, respectively), \%AceCOX-1 in apparently normal rectal mucosal biopsies averaged $61 \pm 9 \%$ and $66 \pm 8 \%$, respectively.

AceCOX-1 was also detected in biopsies of the left and right colon in both groups (Figure 2); \%AceCOX-1 values were not significantly different from the values found in the rectal mucosa. The values of \%AceCOX-1 in colorectal mucosa were 
(a)

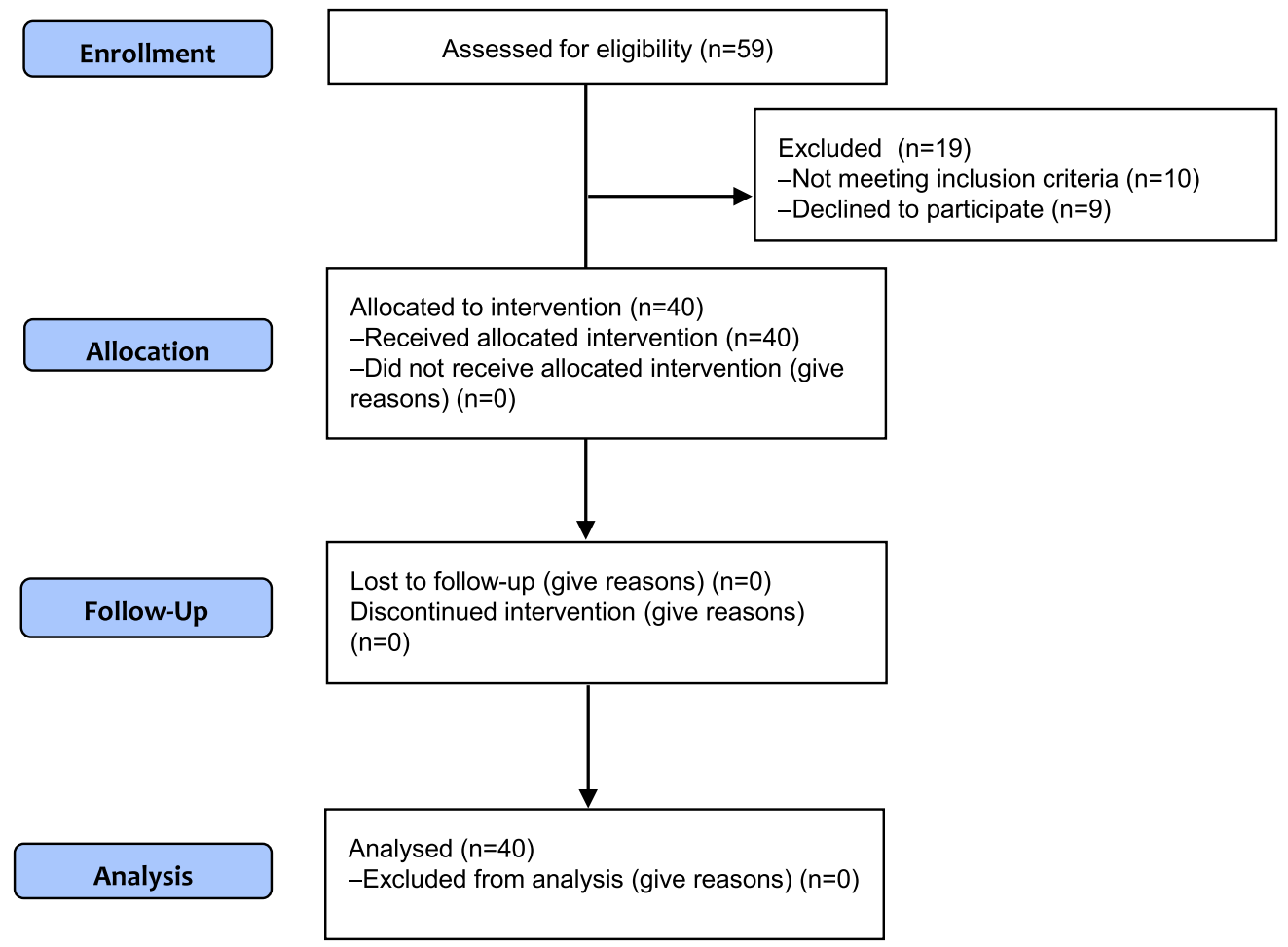

(b) Enteric-coated low dose aspirin, 100mg once daily

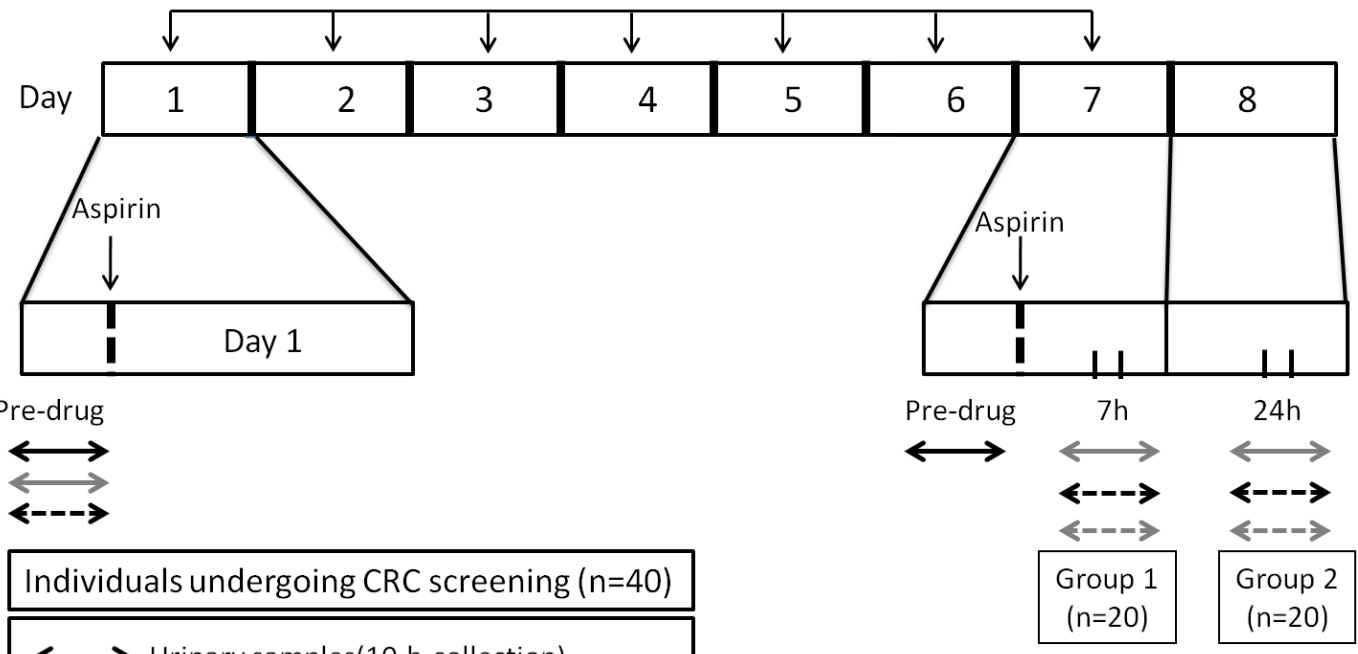

$\longleftrightarrow$ Urinary samples(10 h-collection)

$\longleftrightarrow$ Blood samples

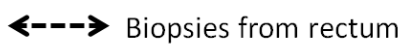

$\leftrightarrow--->$ Biopsies from left and right colon

Figure 1 Flow diagram of the study phases (a) and flow chart of the study protocol (b). [Color figure can be viewed at wileyonlinelibrary.com]

significantly lower $(P<0.01)$ than those measured in platelets, both at 7 and $24 \mathrm{~h}$ after dosing.

\section{Effects of low-dose aspirin on rectal mucosal expression of} proteins involved in the pathophysiology of colorectal cancer In addition to the proteins of the enzymatic machinery responsible for $\mathrm{PGE}_{2}$ biosynthesis and catabolism, we assessed the impact of aspirin treatment on rectal mucosal levels of EGFR (epidermal growth factor receptor) ${ }^{25}$ and S6. EGFR levels were not affected by the drug (Supplementary Figure S1). Similarly, average values of S6 levels detected in both groups were not significantly affected by low-dose aspirin (Figure 4a); however, some changes were detected in some individuals of group 1 (Figure $\mathbf{4 b}$ ) and group 2 (not shown). The drug caused a significant reduction $(P<0.05)$ 
Table 1 Antiplatelet effects of low-dose aspirin evaluated using direct and indirect biomarkers of drug action

\begin{tabular}{lccc}
\hline & Group 1 & Group 2 & $P$ \\
\hline AceCOX-1(\%) & $74.8 \pm 8.1$ & $75.2 \pm 8.8$ & 0.899 \\
\hline Serum TXB 2 (\% inhibition) & $97.4 \pm 2.8$ & $97.6 \pm 1.4$ & 0.761 \\
\hline PFA-100 (CEPI-CT, \% of baseline) & $205.4 \pm 52.9$ & $200.0 \pm 58.5$ & 0.772 \\
\hline TX-M (\% inhibition) & $68.0 \pm 13.8$ & $69.0 \pm 16.5$ & 0.837
\end{tabular}

Values are mean $\pm \mathrm{SD}, n=20$ /group; $P$ values are for the mean comparisons of group 1 vs. group 2.

in the phosphorylation of S6 (p-S6), in both groups (Figure $\mathbf{4 b , c}$ ). Bivariate linear regression analysis showed that the values of rectal mucosal p-S6/S6 significantly $(P<0.01)$ correlated with the levels of $\mathrm{PGE}_{2}$, assessed in the same samples (Figure $\mathbf{4 d}$ ).

\section{Effect of low-dose aspirin on systemic biosynthesis of $\mathrm{PGE}_{2}$ and $\mathrm{PGI}_{2}$}

Our population included 19 individuals (47.5\%) with polyps/ colorectal adenomas/adenocarcinomas (Supplementary Table S1). Thus, we studied whether the systemic biosynthesis of $\mathrm{PGE}_{2}$ is altered in this setting by assessing the urinary levels of PGE-M, a major enzymatic metabolite of $\mathrm{PGE}_{2}{ }^{26}$ Median (range) baseline values of urinary PGE-M excretion were 10.6 (4.4-48.7) ng/mg creatinine (Figure 5a). Multivariate regression analysis showed that none of the demographic and clinical variables and drug treatments made a significant contribution to baseline PGE-M levels. In the 19 individuals with polyps/adenomas/adenocarcinomas, median values of PGE-M were substantially unchanged: 10.0 (4.6-48.7) $\mathrm{ng} / \mathrm{mg}$ creatinine (not shown). After dosing with low-dose aspirin, median urinary PGE-M excretion measured in both groups was $9.3(3.6-30.2) \mathrm{ng} / \mathrm{mg}$ creatinine (Figure 5a). The use of a nonparametric paired analysis showed that aspirin caused a significant $(P<0.05)$ reduction in PGE-M excretion vs. baseline (Figure 5a).

Low-dose aspirin did not significantly affect the urinary levels of PGI-M, a major enzymatic metabolite of prostacyclin mainly derived from vascular COX-27 ${ }^{27}$ (Figure 5b).

\section{Effects of aspirin on intestinal epithelial COX-1 and COX-2 and platelet COX-1 activities in vitro}

In quiescent human intestinal epithelial cells (expressing COX-1, but not COX-2: Figure 6a), aspirin inhibited $\mathrm{PGE}_{2}$ production in a concentration-dependent fashion, with an $\mathrm{IC}_{50}$ value of $8.3 \mu \mathrm{mol} / \mathrm{L}$ (95\% confidence interval, CI, 4.6-15.2) (Figure 6b). The inhibitory effect of aspirin on COX-2-dependent $\mathrm{PGE}_{2}$ was assessed in cells expressing COX-2 following their incubation with $2 \mathrm{ng} / \mathrm{mL}$ of interleukin-1 beta (IL-1 $\beta$ ) for $12 \mathrm{~h}$ (Figure 6a). Under these experimental conditions, aspirin inhibited $\mathrm{PGE}_{2}$ production, in a concentration-dependent fashion, with an $\mathrm{IC}_{50}$ value of $12.0 \mu \mathrm{mol} / \mathrm{L}$ (95\% CI, 6.9-22.8) (Figure 6b). Finally, we characterized the potency of aspirin in inhibiting $\mathrm{TXB}_{2}$ production in human platelets. In washed platelets (expressing only COX-1, Supplementary Figure S2), aspirin reduced platelet
$\mathrm{TXB}_{2}$ production, in a concentration-dependent fashion, with an $\mathrm{IC}_{50}$ value of $8.2 \mu \mathrm{mol} / \mathrm{L}$ (95\% CI, 4.8-14.1) (Figure 6b).

\section{Stability and rate of de novo synthesis of COX-1 and COX-2 in human intestinal epithelial cells in vitro}

In the presence of the protein synthesis inhibitor cycloheximide (CHX, $50 \mu \mathrm{mol} / \mathrm{L}$ final concentration), COX-1 protein levels remained stable up to $24 \mathrm{~h}$ (Figure 6c) while COX-2 levels, induced in response to IL-1 $\beta$, decreased in a time-dependent fashion with a $t_{1 / 2}$ of $\sim 3 \mathrm{~h}$ (Figure $6 \mathrm{c}$ ).

We studied the rate of recovery of $\mathrm{PGE}_{2}$ biosynthesis in epithelial cells (expressing only COX-1) and in a human colonic adenocarcinoma cell line (HCA7 cells, which constitutively express only COX-2, Supplementary Figure S2), after treatment with aspirin $(50 \mu \mathrm{mol} / \mathrm{L})$ for $1 \mathrm{~h}$ followed by drug washout. At $24 \mathrm{~h}$ after aspirin exposure, $\sim 50 \%$ of COX-1 activity was recovered, while COX-2 activity was almost completely restored (Figure 6d).

\section{Effect of repeated exposure to aspirin on epithelial coX-1-dependent $\mathrm{PGE}_{2}$ production in vitro}

Epithelial cells were exposed for $1 \mathrm{~h}$ to aspirin $(2.5 \mu \mathrm{mol} / \mathrm{L})$ and the drug was washed away from the cells; this was repeated once daily up to $72 \mathrm{~h}$. The activity of COX-1 was induced by the incubation with arachidonic acid (AA) $(20 \mu \mathrm{mol} / \mathrm{L})$ for $60 \mathrm{~min}$. As shown in Figure 6e, a cumulative inhibition of $\mathrm{PGE}_{2}$ production was caused by repeated exposure of epithelial cells to a low concentration of aspirin.

\section{DISCUSSION}

Several lines of evidence support a chemopreventive effect of aspirin against CRC. ${ }^{1-3}$ However, prevention of sporadic colorectal polyp recurrence represents the only clinical setting in which the chemopreventive effects of low- to medium-dose aspirin and

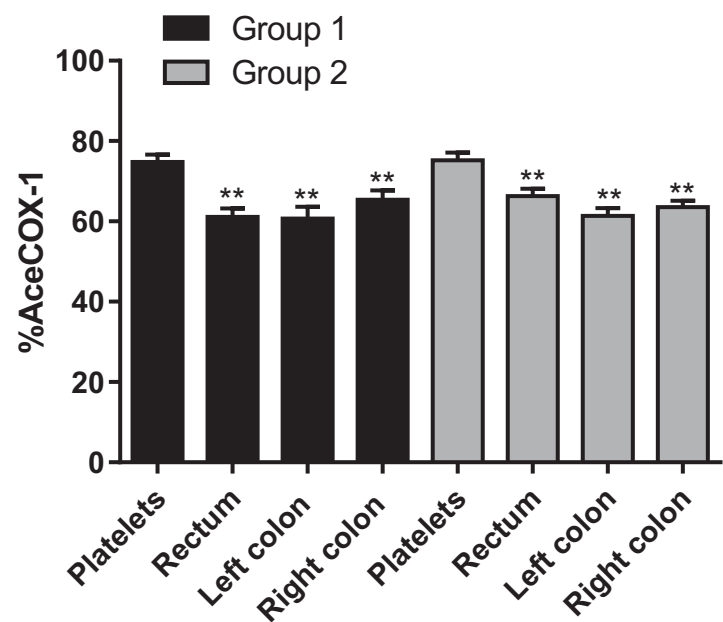

Figure 2 Low-dose aspirin acetylates COX-1 in platelets and apparently normal colorectal mucosa. Using an LC-MS/MS technique, acetylated COX-1 vs. total COX-1 (\%AceCOX-1) in homogenized platelets and biopsies collected from the rectum and the left and right colon of group 1 and group 2 (at 7 and $24 \mathrm{~h}$ following the last aspirin dose, respectively) was assessed. Data are reported as mean \pm SEM, $n=20$ /group. $* * P<0.01$ vs. platelets. 
(a)

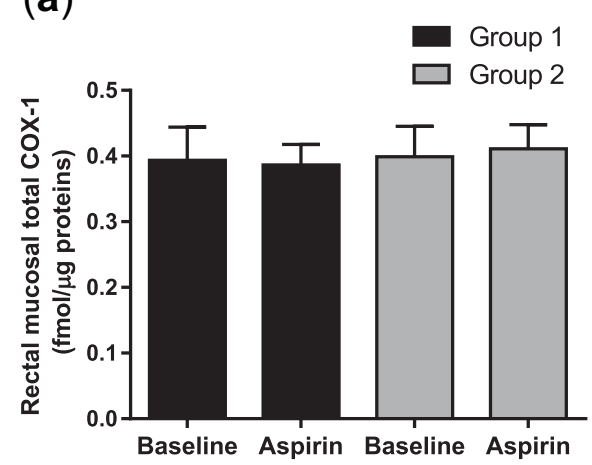

(b)

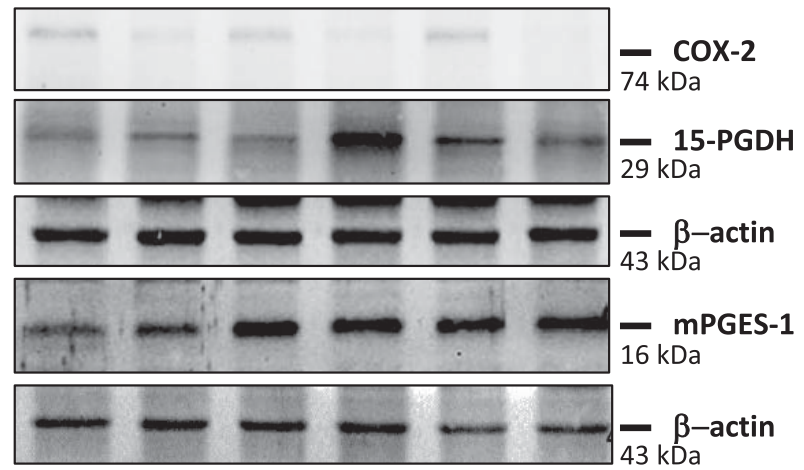

Baseline Aspirin Baseline Aspirin Baseline Aspirin

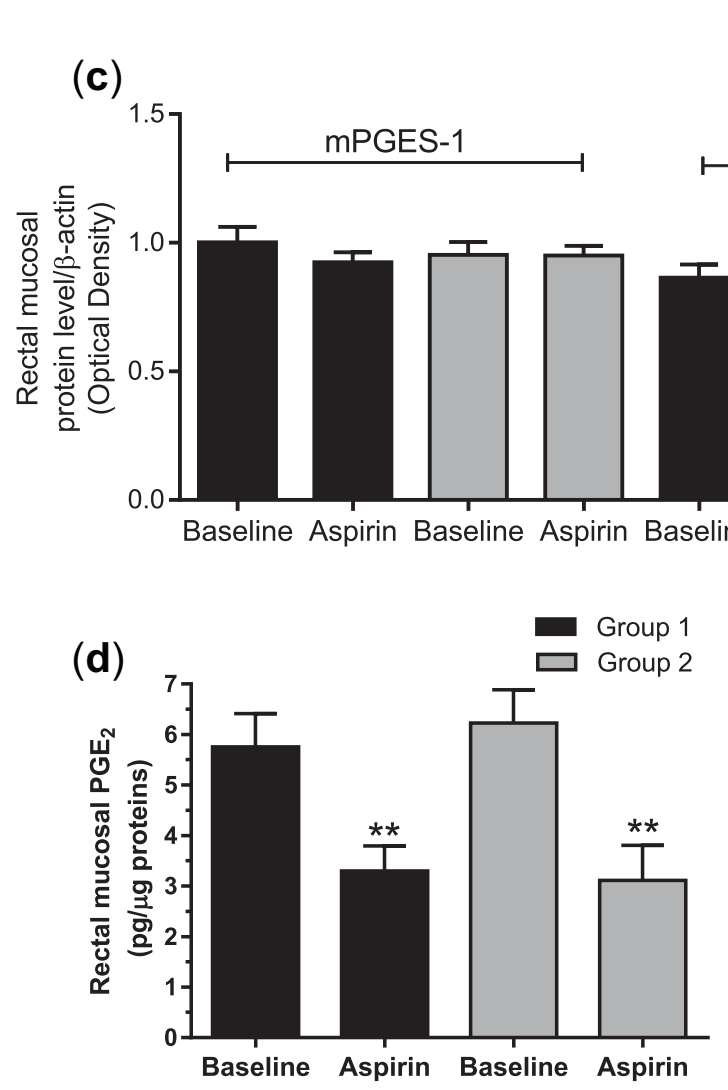

Figure 3 Effects of low-dose aspirin on the levels of COX-1, COX-2, mPGES-1, 15-PGDH, and PGE 2 , in apparently normal rectal mucosal biopsies. In rectal mucosal samples collected at 7 and $24 \mathrm{~h}$ after the last dose of aspirin (groups 1 and 2, respectively), we evaluated (a) COX-1 concentrations (AceCOX$1+\mathrm{COX}-1$ ) (fmol/ $\mu$ g protein) by LC-MS/MS, (b) COX-2, mPGES-1, 15-PGDH, and $\beta$-actin protein levels (by western blot, the analysis of three representative biopsies collected in different individuals of group 1 is shown), (c) densitometric analysis of western blot bands of mPGES-1 and 15-PGDH normalized to $\beta$-actin, (d) $\mathrm{PGE}_{2}$ levels (pg/ $\mu$ g protein; by immunoassay). Data are reported as mean $\pm \mathrm{SEM}, n=20 /$ group (a,c,d). $* * P<0.01$ vs. baseline (d).

medium- to high-dose coxibs (selective COX-2 inhibitors) have been examined prospectively in placebo-controlled randomized trials. ${ }^{3,28}$ The results of these trials demonstrate that: 1 ) the chemopreventive effect of aspirin is apparent at daily doses as low as $81 \mathrm{mg} ; 2)$ it does not display dose-dependence in a head-to-head dose comparison; and 3) a protective effect against colorectal tumorigenesis is also found with coxibs. ${ }^{3}$ The current knowledge of the human pharmacodynamics of low-dose aspirin (i.e., a selective inhibitor of platelet COX-1 activity) and coxibs (i.e., selective inhibitors of COX-2 activity) supports the hypothesis that both COX-isozymes contribute to intestinal tumorigenesis. ${ }^{3,29,30}$ It has been proposed that COX-1-dependent platelet activation, occurring at sites of persistent mucosal injury, may participate in COX-2 induction in stromal and epithelial cells through the 
(a)

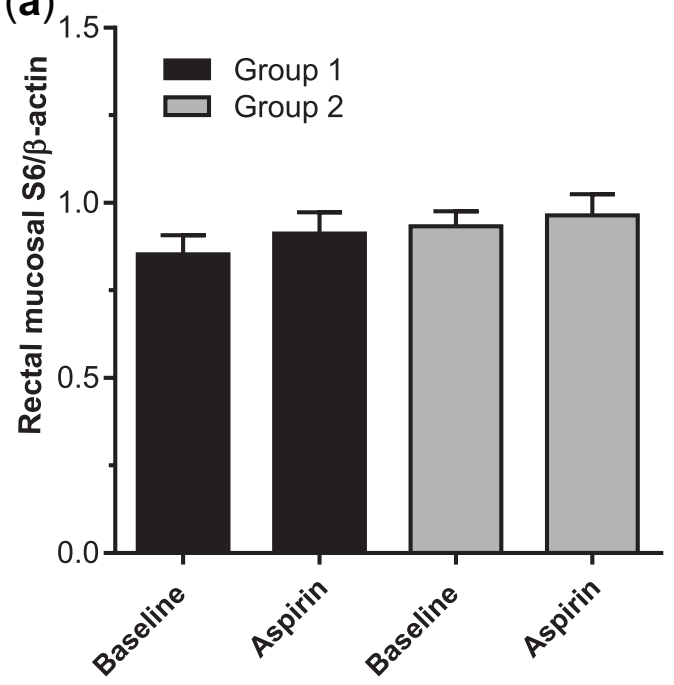

(c)

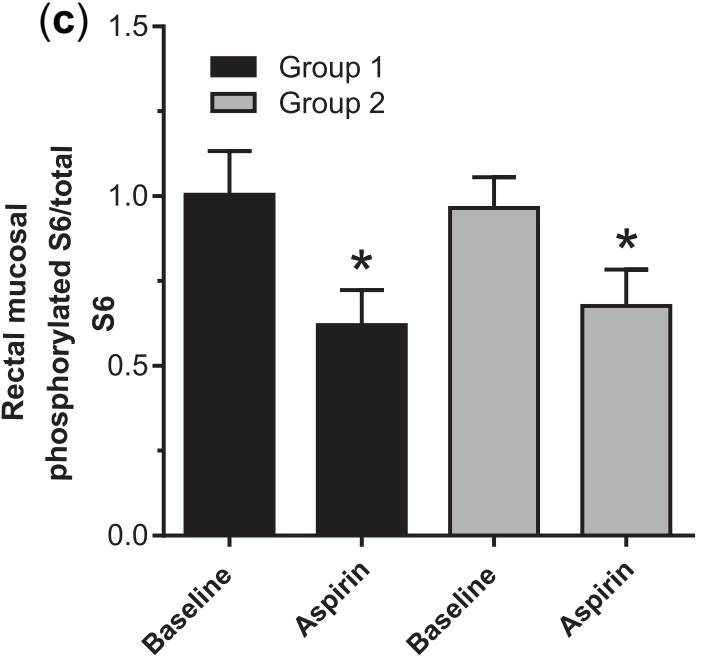

(b)

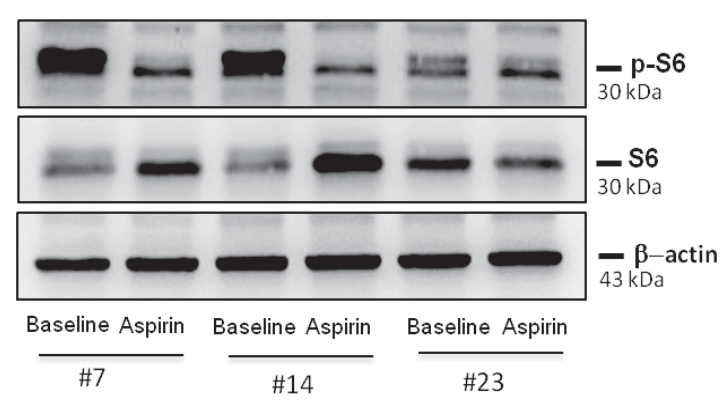

(d)

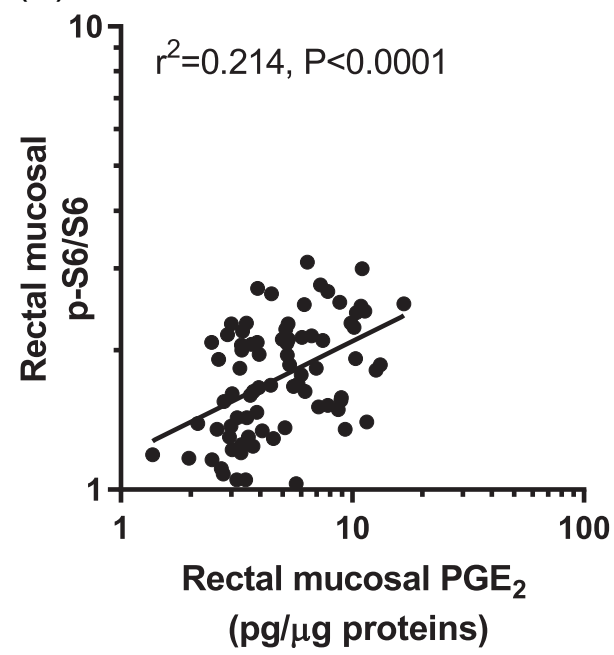

Figure 4 Effects of low-dose aspirin on the rectal mucosal levels of S6 and its phosphorylation. (a,c) Quantification of S6 and its phosphorylated form p-S6 in rectal mucosa of all individuals (groups 1 and 2, samples collected at 7 and $24 \mathrm{~h}$ after the last aspirin dose, respectively) by western blot (normalized to $\beta$-actin values); baseline is predrug and Aspirin is postdrug. (b) A representative western blot analysis of biopsies collected from three

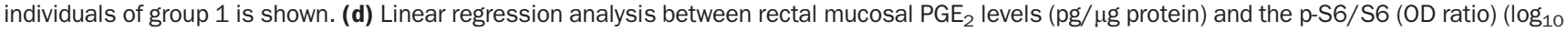
transformation of values) measured in the same samples. Data are reported as mean $\pm \mathrm{SEM}, n=20 /$ group $(a, b)$. $* P<0.05$ vs. baseline (b).

release of a plethora of soluble mediators (i.e., lipids, cytokines, and growth factors) and membrane vesicles (rich in genetic material, including microRNAs). ${ }^{29}$ This hypothesis would explain the apparently similar chemopreventive effect of low-dose aspirin acting upstream to suppress platelet activation at sites of intestinal mucosal injury and coxibs acting downstream to inhibit COX-2 activity in stromal and intestinal epithelial cells.

However, this interpretation remains highly hypothetical in the absence of a quantitative assessment of platelet vs. extraplatelet COX-1 acetylation by low-dose aspirin.

Using a novel proteomic assay that allows assessing the extent of acetylation of COX-1 at Ser529 in cells/biopsies, ${ }^{20,23}$ we were able to demonstrate that low-dose aspirin can cause a persistent acetylation of constitutively expressed COX-1 in rectal and left and right colonic mucosa.
Low-dose aspirin was more effective in acetylating COX-1 in blood platelets than in colorectal mucosa, despite inhibiting COX-1 activity with comparable potency in intestinal epithelial cells and platelets in vitro (refs. ${ }^{31,32}$, and the present study). The exposure of platelets to a higher concentration of intact ASA in the presystemic circulation ${ }^{33}$ is a plausible determinant of the higher extent of acetylation of COX-1 in platelets than colorectal mucosa demonstrated in the present study.

Acetylation of rectal mucosal COX-1 by low-dose aspirin was associated with an $\sim 50 \%$ reduction in $\mathrm{PGE}_{2}$ levels, which persisted up to $24 \mathrm{~h}$ after dosing. We found that both the stability of COX-1 (up to $24 \mathrm{~h}$ ) and the slow-rate of recovery of COX-1 activity might explain the long-term persistence of enzyme acetylation and product inhibition. Thus, $24 \mathrm{~h}$ after the exposure to an aspirin concentration which causes a substantial inhibition of 

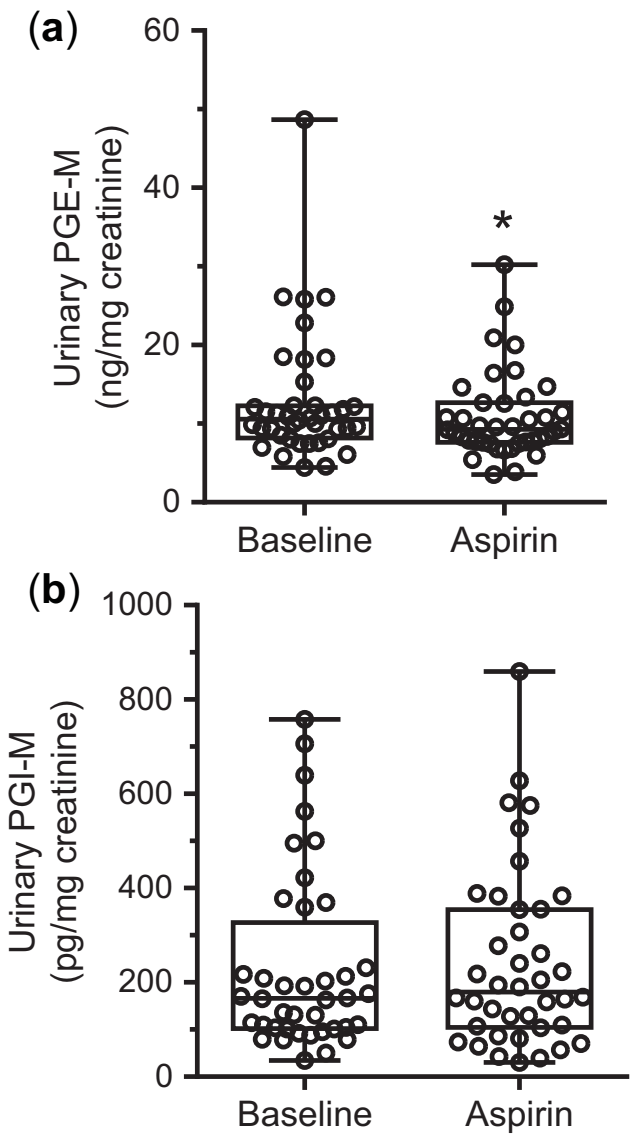

Figure 5 Effect of low-dose aspirin on the systemic biosynthesis of $\mathrm{PGE}_{2}$ and $P \mathrm{Pl}_{2}$. Urinary PGE-M levels (ng/mg creatinine) (a) and PGI-M levels (pg/mg creatinine) (b) at baseline and after low-dose aspirin treatment. Data $(n=40)$ are reported as a box-and-whisker plot with individual data points; the line represents the median value. ${ }^{*} P<0.05$ vs. baseline (a).

COX-1 activity, the enzyme is still profoundly inhibited, and repeated daily exposure to aspirin in vitro is associated with a cumulative inhibition of $\mathrm{PGE}_{2}$ production. This is analogous to the cumulative inhibition of platelet $\mathrm{TXB}_{2}$ production upon repeated daily dosing with very low doses of aspirin. ${ }^{34}$

In the present study, rectal mucosal $\mathrm{PGE}_{2}$ levels correlated with the extent of phosphorylation of $\mathrm{S} 6$ and low-dose aspirin caused a significant reduction in the phosphorylation of the protein.

Din et al. ${ }^{35}$ previously reported that aspirin reduces $S 6$ phosphorylation in CRC cells in vitro at millimolar concentrations. Moreover, rectal mucosal samples from patients given aspirin $600 \mathrm{mg}$ daily for 1 week had reduced phosphorylation of S6. ${ }^{35}$ The authors concluded that aspirin is an inhibitor of mTOR and an activator of AMPK, targeting regulators of intracellular energy homeostasis and metabolism. Here we report that the administration of low-dose aspirin caused a significant reduction in rectal mucosal S6 phosphorylation at $7 \mathrm{~h}$ after dosing, at a time of circulating micromolar concentrations of ASA. The persistence of this effect up to $24 \mathrm{~h}$ after dosing (when no levels of ASA were detectable) is consistent with aspirin acting by an irreversible mechanism. Together with the significant relationship of rectal mucosal p-S6/S6 values with $\mathrm{PGE}_{2}$ levels, these results suggest that $\mathrm{COX}$-1-dependent $\mathrm{PGE}_{2}$ inhibition by low-dose aspirin may explain downstream S6 phosphorylation.

We provide evidence that a low concentration of aspirin is inadequate to cause a persistent inhibitory effect on epithelial $\mathrm{COX}$-2-dependent $\mathrm{PGE}_{2}$ biosynthesis in vitro. In fact, the effect on $\mathrm{PGE}_{2}$ production was completely reversed at $24 \mathrm{~h}$. These results might suggest the inability of low-dose aspirin to cause a cumulative effect upon repeated daily dosing. However, this issue should be investigated in an appropriate study of patients with COX-2-expressing CRC, using a direct marker of aspirin action.

We assessed the impact of low-dose aspirin on indices of COX-2 activity in vivo, such as urinary PGE-M ${ }^{26}$ and PGI-M excretion. $^{27}$ We found a significant $(P<0.05)$ small reduction of median values of urinary PGE-M by low-dose aspirin while urinary PGI-M levels did not differ to any statistically significant extent. These assessments were secondary endpoints and the statistical power of the clinical study was inadequate to detect moderate treatment effects. Recently, Boutaud et al. ${ }^{32}$ reported that low-dose aspirin reduced urinary PGE-M and PGI-M levels by $45 \%$ and $37 \%$, respectively, in a larger population of healthy subjects.

The limitations of our study include: 1) given the nature, duration, and sample size, we could not assess any clinical end-point; 2) in the absence of detectable COX-2 expression in colorectal mucosa biopsies, we could not directly assess the extent and duration of COX-2 acetylation throughout the 24-h dosing interval. The strengths are represented by: i) use of a recently developed proteomic approach to quantitate the extent of COX-1 acetylation $^{20,23}$ in blood and tissue cellular targets of aspirin pharmacodynamics; ii) two randomized groups of subjects, in whom pharmacokinetic/pharmacodynamic assessment was performed shortly after peak plasma levels of intact ASA in the systemic circulation and at $24 \mathrm{~h}$ after dosing; and iii) in vitro experiments to characterize the stability of COX-1 vs. COX-2 protein, and cumulative nature of COX-1 acetylation in colorectal mucosa.

In conclusion, aspirin $100 \mathrm{mg}$ once daily can persistently acetylate intestinal COX-1, thus affecting colorectal mucosa $\mathrm{PGE}_{2}$ biosynthesis and the phosphorylation of S6. Our results suggest that the concurrent inhibitory effect of low-dose aspirin on COX-1 in platelets and colorectal mucosa might explain its preventive efficacy against recurrence of a colorectal adenoma ${ }^{3,28}$ and its longer-term chemopreventive benefit against CRC in the setting of primary cardiovascular prevention. ${ }^{37}$

\section{METHODS}

Clinical study: design, treatments, and biomarker assessment The main objectives of this study were: 1) to verify whether low-dose aspirin causes persistent acetylation of COX-1 in platelets, but not in nucleated cells of the recto-colonic tissue $24 \mathrm{~h}$ after dosing when no circulating levels of ASA are detectable ${ }^{20} ; 2$ ) to assess whether at the time when aspirin reaches its maximal concentration in the systemic circulation $(\sim 7 \mathrm{~h}),{ }^{20} \mathrm{COX}-1$ of platelets and colorectal mucosa is acetylated to a different extent; 3) to evaluate whether differential acetylation of COX-1 translates into complete (in platelets) vs. incomplete (in colorectal mucosa) suppression of prostanoid production. The percentage of COX-1 acetylation at Ser529 (AceCOX-1) in washed platelets and 
(a)
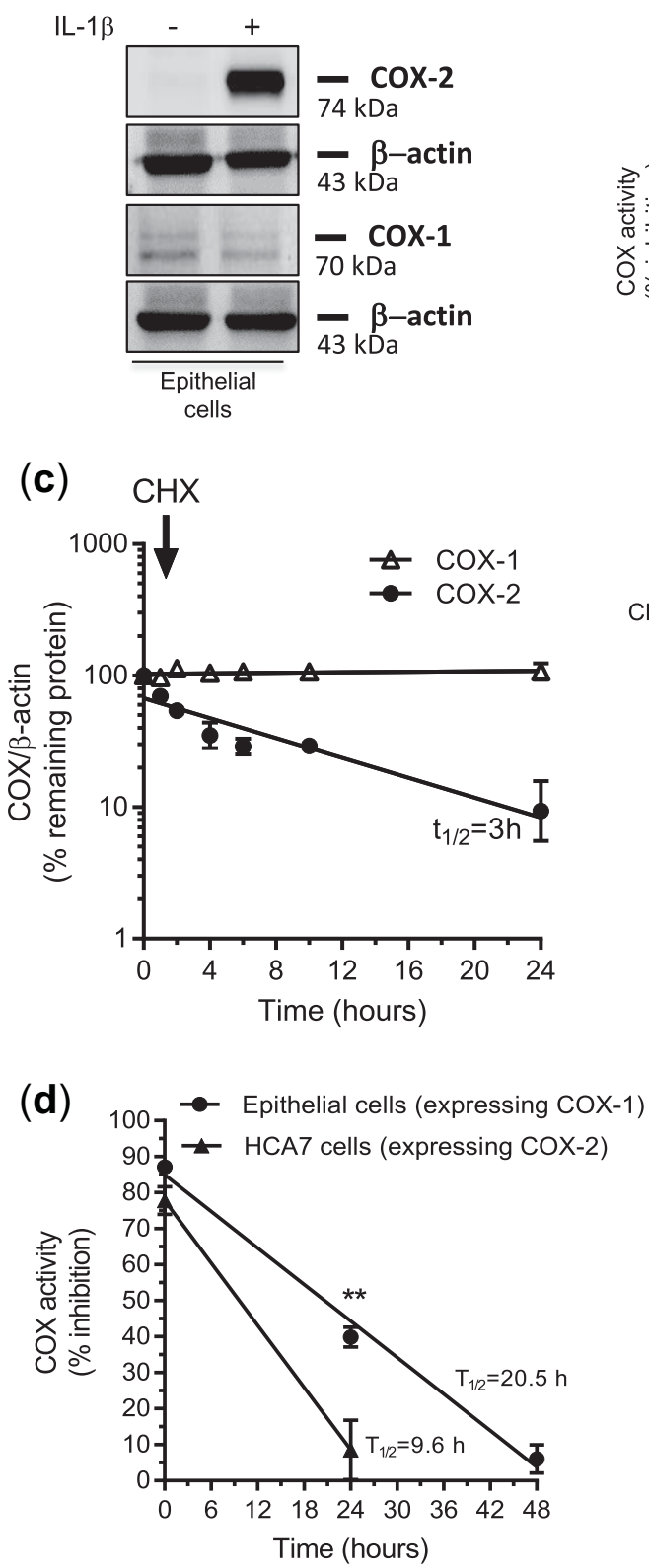

(b)
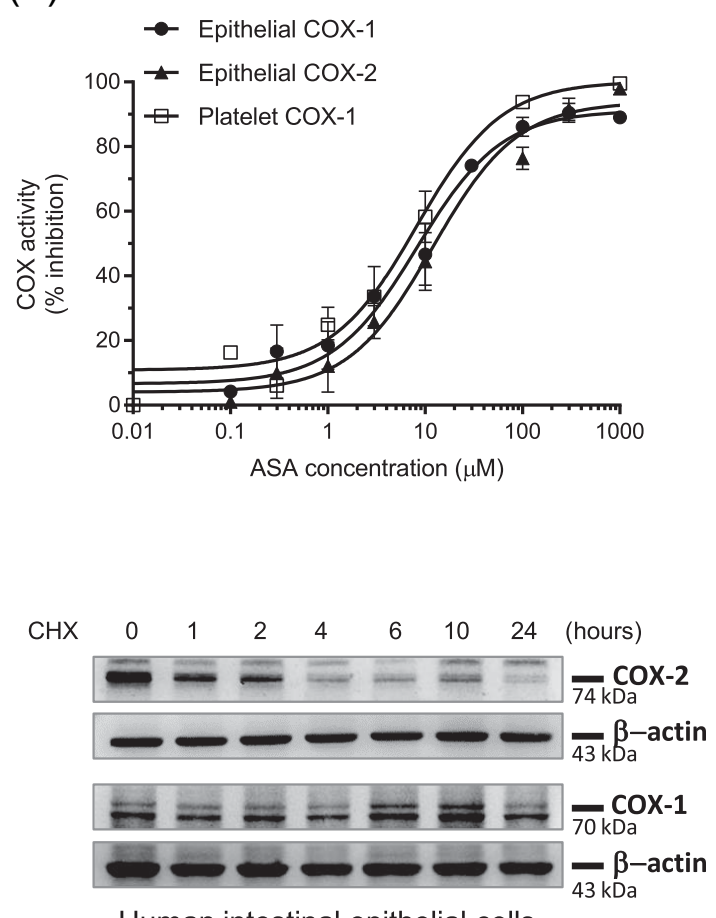

Human intestinal epithelial cells

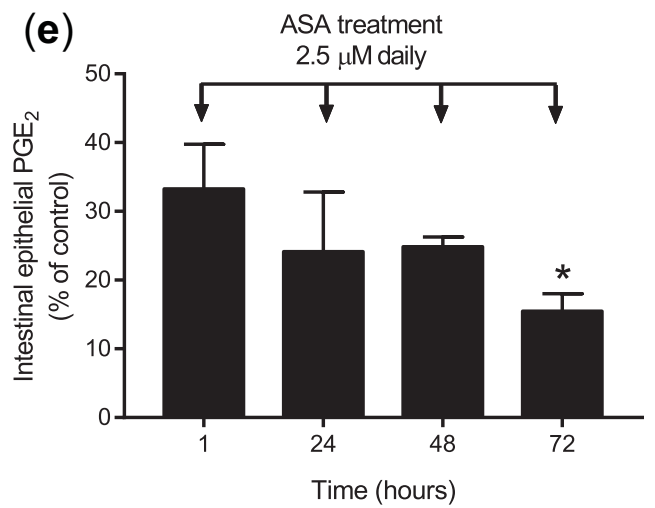

Figure 6 In vitro characterization of aspirin inhibitory effects on COX-isozyme activity and protein stability in epithelial cells and platelets. (a) Analysis of COX-1, COX-2, and $\beta$-actin protein levels in intestinal epithelial cells (untreated or stimulated with IL-1 $(2 \mathrm{ng} / \mathrm{mL})$ for $12 \mathrm{~h}$ ) by western blot. (b) Concentration-dependent inhibitory effect of aspirin (ASA) on COX activity, assessed by measuring PGE 2 levels in untreated intestinal epithelial cells (epithelial COX-1), in IL-1 $\beta$-stimulated intestinal epithelial cells (epithelial COX-2), and TXB 2 levels in washed platelets (platelet COX-1). (c) The stability of COX-1 and COX-2 proteins, in untreated and IL-1 $\beta$-stimulated intestinal epithelial cells, respectively, was assessed in the presence of cycloheximide (CHX; $50 \mu \mathrm{mol} / \mathrm{L}$ final concentration) for the indicated times and then cell lysates were prepared and analyzed by western blotting for COX-1, COX-2, and $\beta$-actin. (d) Time-dependent recovery of COX-1 and COX-2 activity by aspirin-treated intestinal epithelial cells and HCA7 cells, respectively; cells were treated for $1 \mathrm{~h}$ with aspirin $50 \mu \mathrm{mol} / \mathrm{L}$, then the cells were washed to remove aspirin and, at the indicated times, percent inhibition of COX-1 and COX-2

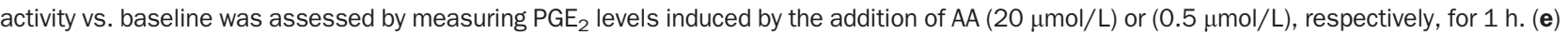
Cumulative inhibition of epithelial cell COX-1 activity by daily exposure to very low concentrations of aspirin (2.5 $\mu \mathrm{mol} / \mathrm{L}$ ) or vehicle (DMSO) for $1 \mathrm{~h}$; then the drug was washed away from the cells and this treatment was repeated once daily up to $72 \mathrm{~h}$ and the activity of COX-1 was assessed as described above. Data are reported as mean \pm SEM, $n=3-7$ (b-e); ** $P<0.01$ vs. $24 \mathrm{~h}$ for COX-2 (d); *P $<0.05$ vs. 1 h (e).

colorectal mucosa biopsies was assessed by the absolute quantification (AQUA) method using an LC-MS/MS technique, as previously reported $^{20,23}$ and briefly described in the Supplementary Methods. The impact of low-dose aspirin treatment was evaluated on: 1) platelet COX-1 activity, by assessing serum $\mathrm{TXB}_{2}$ levels ${ }^{19,20,34}$; 2) platelet function by using the whole-blood PFA-100 as previously described ${ }^{20}$; 3) $\mathrm{PGE}_{2}$ biosynthesis assessed by a validated immunoassay ${ }^{38}$ and the expression of proteins involved in CRC development (COX-1, COX-2, mPGES-1, 15-PGDH, EGFR, and S6, both native protein and phosphorylated form $\left.\left(\mathrm{p}-\mathrm{S} 6^{\text {Ser235/236 }}\right)\right)$ in apparently normal rectal tissues by 
western blot, as detailed in the Supplementary Methods. Finally, we assessed the impact of low-dose aspirin on biomarkers of the in vivo activity of COX-2, by assessing the urinary excretion of major enzymatic metabolites of $\mathrm{PGE}_{2}$ and $\mathrm{PGI}_{2}$, i.e., 11- $\alpha$-hydroxy-9,15-dioxo-2,3,4,5tetranor-prostane-1,20-dioic acid (PGE-M) and 2,3-dinor-6-keto-PGF $1 \alpha$ (PGI-M), respectively, ${ }^{39,40}$ and COX-1 by assessing the urinary excretion of a major enzymatic metabolite of $\mathrm{TXA}_{2}$, i.e., 11-dehydro- $\mathrm{TXB}_{2}$ (TX$\mathrm{M}),{ }^{21,22}$ using previously described methods. ${ }^{2,39,40}$ Due to the complexity of the clinical protocol, urinary samples were collected in all individuals, during the last $10 \mathrm{~h}$ (overnight) after the 6th dose of low-dose aspirin (administered in the morning).

The study was conducted in accordance with the Declaration of Helsinki, and the protocol was approved by the Clinical Investigation Ethics Committee of the Aragón Health Research Institute (Zaragoza, Spain) (EUDRACT number: 2013-004269-15; ClinicalTrials.gov Identifier: NCT02125409). All subjects provided written informed consent.

The study was performed at Hospital Clinico Universitario Lozano Blesa (Zaragoza, Spain) and all biomarker assessments were carried out at CeSI-MeT (Chieti, Italy).

\section{In vitro studies in human intestinal epithelial cells, platelets and human colon cancer cell line HCA-7}

The experimental methods are reported online in the Supplementary Information.

\section{Statistical analysis}

The data are expressed as mean $\pm \mathrm{SD}$, unless otherwise stated, and statistical comparisons were made by parametric tests (Student's $t$-test or repeated-measures analysis of variance followed by the StudentNewman-Keuls test). Data that did not pass the normality test (by the method of Kolmogorov and Smirnov) are reported as median and range (minimum and maximum) and analyzed using nonparametric tests. A probability value of $P<0.05$ was considered statistically significant. The primary endpoint of the study was the assessment of COX-1 acetylation in platelets vs. nucleated cells of the colonic tissue, at $24 \mathrm{~h}$ after dosing. The primary hypothesis was that the administration of EC-aspirin would cause a maximal acetylation of platelet COX-1 ( 70\%) while causing a marginal acetylation $(<10 \%)$ of COX-1 in colonic tissue, at $24 \mathrm{~h}$ after dosing (group 2). The secondary hypothesis was that after $7 \mathrm{~h}$ of aspirin administration (group 1), the drug would cause maximal acetylation of platelet COX-1 associated with $20-30 \%$ COX-1 acetylation in intestinal tissue. Based on a previous study performed in healthy subjects, we calculated an intersubject coefficient of variation (CV) of platelet AceCOX-1 of $10 \%$, at $24 \mathrm{~h}$ after aspirin administration for 7 days. ${ }^{20}$ Assuming this intersubject $\mathrm{CV}$, a sample size of 20 patients would allow detection of a difference of $14 \%$ or higher in percent acetylation between platelets vs. colonic tissues, with a power of $99 \%$, by two-tailed tests, with probability values less than the type I error rate of 0.05 . Thus, we would be able to obtain significant differences of AceCOX-1 in platelets vs. colonic tissue both at 24 (group 2) and at $7 \mathrm{~h}$ (group 1) after dosing.

Additional Supporting Information may be found in the online version of this article.

\section{ACKNOWLEDGMENTS}

We thank Valentina Olivastri, PharmD, Gianluca Grimaldi, PharmD, and Rosalia Grande, PharmD (“G. d'Annunzio” University) and the personnel of IIS Aragon and University Clinic Hospital Lozano Blesa in Zaragoza ( $\mathrm{Dr}$ R. Cornudella, P. Roncales, A Ferrandez, F. Sopeña, E. Chueca, and S. Arechavaleta) for their generous cooperation.

\section{CONFLICT OF INTEREST/DISCLOSURE}

A. Lanas reports grants and nonfinancial support from Bayer Pharma AG during the conduct of the study and personal fees from Bayer outside the submitted work. C. Patrono received consultant and speaker fees from AstraZeneca and Bayer. P. Patrignani reports personal fees from Bayer during the conduct of the study. The other authors declare no conflicts of interest. This was an investigator-initiated study and Bayer Pharma AG provided support to the principal investigator (A. Lanas) by supplying ECaspirin and some financial resources. Bayer did not play any role in the design of the protocol, in the interpretation of the results, or in the decision to publish them. This work was also supported by the Ministero dell'Istruzione, dell'Università e della Ricerca (MIUR) (Grant PRIN 20102011, protocol number 2010FHH32M), and Associazione Italiana per la Ricerca sul Cancro (Grant IG-12111) (to P. Patrignani) and Instituto de Salud Carlos III, Spain (PI14/01218) (to A. Lanas).

\section{AUTHOR CONTRIBUTIONS}

P.P., A.L., and C.P. wrote the article; P.P., A.L., and C.P. designed the research; A.S., C.S., E.P., M.D., S.T., A.C., A.B., S.S., L.D.F., and M.Z. performed the research; P.P. and A.L. analyzed the data.

(c) 2017 American Society for Clinical Pharmacology and Therapeutics

1. Rothwell, P.M. et al. Effect of daily aspirin on risk of cancer metastasis: a study of incident cancers during randomised controlled trials. Lancet 379, 1591-1601 (2012).

2. Rothwell, P.M. et al. Short-term effects of daily aspirin on cancer incidence, mortality, and non-vascular death: analysis of the time course of risks and benefits in 51 randomised controlled trials. Lancet 379, 1602-1612 (2012).

3. Patrignani, P. \& Patrono C. Aspirin and cancer. J. Am. Coll. Cardiol. 68, 967-976 (2016).

4. Patrignani, P. \& Patrono, C. Cyclooxygenase inhibitors: From pharmacology to clinical read-outs. Biochim. Biophys. Acta. 1851 422-432 (2015).

5. Dovizio, M., Bruno, A., Tacconelli, S. \& Patrignani, P. Mode of action of aspirin as a chemopreventive agent. Recent Results Cancer Res. 191, 39-65 (2013).

6. Loll, P.J., Picot, D. \& Garavito, R.M. The structural basis of aspirin activity inferred from the crystal structure of inactivated prostaglandin H2 synthase. Nat. Struct. Biol. 2, 637-643 (1995).

7. Wang, D. \& Dubois, R.N. The role of COX-2 in intestinal inflammation and colorectal cancer. Oncogene 29, 781-788 (2010).

8. Chulada, P.C. et al. Genetic disruption of Ptgs-1, as well as Ptgs-2, reduces intestinal tumorigenesis in Min mice. Cancer Res. 60, 47054708 (2000).

9. Smartt, H.J. et al. $\beta$-catenin represses expression of the tumour suppressor 15-prostaglandin dehydrogenase in the normal intestinal epithelium and colorectal tumour cells. Gut 61, 1306-1314 (2012).

10. Roth, G.J., Machuga, E.T. \& Ozols, J. Isolation and covalent structure of the aspirin-modified, active-site region of prostaglandin synthase. Biochemistry 22, 4672-4675 (1983).

11. Roth, G.J., Stanford, N. \& Majerus, P.W. Acetylation of prostaglandin synthase by aspirin. Proc. Natl. Acad. Sci. U. S. A. 72, 3073-3076 (1975).

12. Lee, M., Cryer, B. \& Feldman, M. Dose effects of aspirin on gastric prostaglandins and stomach mucosal injury. Ann. Intern. Med. 120, 184-189 (1994).

13. Barnes, C.J., Hamby-Mason, R.L., Hardman, W.E., Cameron, I.L., Speeg, K.V. \& Lee, M. Effect of aspirin on prostaglandin E2 formation and transforming growth factor alpha expression in human rectal mucosa from individuals with a history of adenomatous polyps of the colon. Cancer Epidemiol. Biomarkers Prev. 8, 311-315 (1999).

14. Sample, D. et al. A dose-finding study of aspirin for chemoprevention utilizing rectal mucosal prostaglandin $\mathrm{E}(2)$ levels as a biomarker. Cancer Epidemiol. Biomarkers Prev. 11, 275-279 (2002).

15. Ismail, H.M.S. Downstream the mTOR: S6 kinases between divergence and redundancy. J. Biochem. Pharmacol. Res. 1, 94-105 (2013).

16. Okunishi, K. et al. Inhibition of protein translation as a novel mechanism for prostaglandin $\mathrm{E}_{2}$ regulation of cell functions. FASEB J. 28, 56-66 (2014).

17. Moore, C.E. et al. Identification of CAMP-dependent kinase as a third in vivo ribosomal protein $\mathrm{S} 6$ kinase in pancreatic beta-cells. J. Mol. Biol. 389,480-494 (2009). 
18. Ruvinsky, I. et al. Ribosomal protein S6 phosphorylation is a determinant of cell size and glucose homeostasis. Genes Dev. 19, 2199-2211 (2005).

19. Patrono, C. et al. Low-dose aspirin and inhibition of thromboxane $B_{2}$ production in healthy subjects. Thromb. Res. 17, 317-327 (1980)

20. Patrignani, P. et al. Reappraisal of the clinical pharmacology of lowdose aspirin by comparing novel direct and traditional indirect biomarkers of drug action. J. Thromb. Haemost. 12, 1320-1330 (2014).

21. Catella, F. \& FitzGerald, G.A. Paired analysis of urinary thromboxane $\mathrm{B}_{2}$ metabolites in humans. Thromb. Res. 47, 647-656 (1987).

22. Ciabattoni, G., Maclouf, J., Catella, F., FitzGerald, G.A. \& Patrono, C Radioimmunoassay of 11-dehydrothromboxane B2 in human plasma and urine. Biochim. Biophys. Acta. 918, 293-297 (1987).

23. Li, X. et al. Differential impairment of aspirin-dependent platelet cyclooxygenase acetylation by nonsteroidal antiinflammatory drugs. Proc. Natl. Acad. Sci. U. S. A. 111, 16830-16835 (2014).

24. Boughton-Smith, N.K., Hawkey, C.J. \& Whittle, B.J.R. Biosynthesis of lipoxygenase and cyclo-oxygenase products from $\left[{ }^{14} \mathrm{C}\right]$-arachidonic acid by human colonic mucosa. Gut 24, 1176-1182 (1983).

25. Patrignani, P. \& Dovizio, M. COX-2 and EGFR: Partners in crime split by aspirin. EBioMedicine 2, 372-373 (2015).

26. Wang, D. \& DuBois, R.N. Urinary PGE-M: a promising cancer biomarker. Cancer Prev. Res. 6, 507-510 (2013).

27. McAdam, B.F., Catella-Lawson, F., Mardini, I.A., Kapoor, S., Lawson, J.A. \& FitzGerald GA. Systemic biosynthesis of prostacyclin by cyclooxygenase (COX)-2: the human pharmacology of a selective inhibitor of COX-2. Proc. Natl. Acad. Sci. U. S. A. 96, 272-277 (1999)

28. Cole, B.F. et al. Aspirin for the chemoprevention of colorectal adenomas: meta-analysis of the randomized trials. J. Natl. Cancer Inst. 101, 256-266 (2009).

29. Patrono, C., Patrignani, P. \& García Rodríguez, L.A. Cyclooxygenaseselective inhibition of prostanoid formation: transducing biochemical selectivity into clinical read-outs. J. Clin. Invest. 108, 7-13 (2001).
30. Di Francesco, L., López Contreras, L.A., Sacco, A. \& Patrignani, P. New insights into the mechanism of action of aspirin in the prevention of colorectal neoplasia. Curr. Pharm. Des. 21, 5116-5126 (2015).

31. Bala, M. Acetylation of prostaglandin $\mathrm{H} 2$ synthases by aspirin is inhibited by redox cycling of the peroxidase. Biochem. Pharmacol. 75, 1472-1481 (2008).

32. Boutaud, O. et al. Inhibition of the biosynthesis of prostaglandin E2 by low-dose aspirin: implications for adenocarcinoma metastasis. Cancer Prev. Res. (Phila). 9, 855-865 (2016).

33. Pedersen, A.K. \& FitzGerald, G.A. Dose-related kinetics of aspirin. Presystemic acetylation of platelet cyclooxygenase. N. Engl. J. Med. 311, 1206-1211 (1984)

34. Patrignani, P., Filabozzi, P. \& Patrono, C. Selective cumulative inhibition of platelet thromboxane production by low-dose aspirin in healthy subjects. J. Clin. Invest. 69, 1366-1372 (1982).

35. Din, F.V. et al. Aspirin inhibits mTOR signaling, activates AMP activated protein kinase, and induces autophagy in colorectal cancer cells. Gastroenterology 142, 1504-1515 (2012).

36. Bibbins-Domingo, K., U.S. Preventive Services Task Force. Aspirin use for the primary prevention of cardiovascular disease and colorecta cancer: U.S. Preventive Services Task Force Recommendation Statement. Ann. Intern. Med. 164, 836-845 (2016).

37. Patrignani, P. et al. Biochemical and pharmacological characterization of the cyclooxygenase activity of human blood prostaglandin endoperoxide synthases. J. Pharmacol. Exp. Ther. 271, 1705-1712 (1994).

38. Song, W.L., Lawson, J.A., Wang, M., Zou, H. \& FitzGerald, G.A. Noninvasive assessment of the role of cyclooxygenases in cardiovascular health: a detailed HPLC/MS/MS method. Methods Enzymol. 433, 51-72 (2007)

39. Dovizio, M. et al. Effects of celecoxib on prostanoid biosynthesis and circulating angiogenesis proteins in familial adenomatous polyposis. J. Pharmacol. Exp. Ther. 341, 242-250 (2012). 